\title{
Virtual Resistance Control for Sequential Green-start of Offshore Wind Power Plants
}

\author{
Anubhav Jain (D), Oscar Saborío-Romano (D), Jayachandra N. Sakamuri (D) and Nicolaos A. Cutululis (D)
}

\begin{abstract}
The changing energy landscape due to the large scale integration of renewable energy and shutting down of conventional thermal plants has opened up the potential of alternate sources in the blackstart services market. Grid forming wind turbines can do controlled islanded operation independent of an external grid voltage and thus, participate in network restoration from the start. However, it is necessary to study the capability of wind turbines to deal with the demanding energization transients in a controlled and stable manner. This work investigates the feasibility of using virtual resistance in the wind turbine converter control to reduce transients during self-transformer inrush and sympathetic interaction from downstream string transformers. This can eliminate the need for pre-insertion resistors during sequential energization. The sensitivity of the $\mathrm{AC}$ current and voltage output along with $D C$ link transient to the virtual resistance parameters has also been analyzed using PSCAD simulations. Finally the effectiveness of the proposed method for offshore network energization by a grid forming wind power plant has been tested by comparing to results for a pre-insertion resistor.
\end{abstract}

Index Terms-Wind, HVDC, Grid forming, Energization, Transformer, Inrush, Virtual, Resistance.

\section{INTRODUCTION}

O $\mathrm{NE}$ of the major strategies for sustainable development is the large-scale integration of renewable energy sources in the electrical power system. According to a 2025 renewables forecast [1], the global installed capacity of coal-fired plants is set to peak in 2022 before starting to decline in the following years and be overtaken by solar and wind energy by $13 \%$ in 2025. However as traditional synchronous generators are being replaced with power-electronics interfaced variable generation (like wind and solar), maintaining stable and reliable grid operation becomes more complex due to the changing the power system dynamics [2], [3]. This has increased the risk of wide-area blackouts - for example most recently in South Australia (2016) and UK (2019) - - both initiated by a rapid unexpected decrease in wind power plant generation [4], [5]. Given the changing energy landscape, cost of warming-up large thermal plants and consequently, of blackstart services is increasing. Thus, considerable changes are required to facilitate the participation of alternate sources including aggregated units like large offshore wind power plants (WPP), in the blackstart market [6], [7].

This work is part of the InnoDC project that has received funding from the European Union's Horizon 2020 research and innovation programme under the Marie Skłodowska-Curie grant agreement No 765585.

A. Jain, O. S.-Romano and N. A. Cutululis are with Department of Wind Energy, Technical University of Denmark, 4000 Roskilde, Denmark.

J. N. Sakamuri is with Vattenfall Vindkraft A/S, 6000 Kolding, Denmark.
Many studies have been done on power system restoration with WPPs - a comprehensive review is presented in [8], where it has been highlighted that a change in control philosophy from conventional grid-following to grid-forming (GFM) is essential to facilitate early stage participation of WPPs in bottom-up network energization. Such GFM wind turbines (WT) can operate - albeit with changes to the turbine and converter control [9]-[11] - as a controlled AC voltage source without relying on an external grid and supply load in a power island [8], [12]. This also enables them to provide short term defense against imminent instabilities preceding a blackout by switching to trip-to-houseload and ensuring stable controlled islanded operation (CIO) - potential strategies for restoration procedures in the future decentralized converterrich power system [13].

In addition to reducing the blackout impact, GFM WTs producing power to sustain themselves can avoid health risks, reduce cost of warming and minimize the dependence on backup diesel generator for auxiliary power. This can potentially yield economic benefits, save offshore space, reduce downtime, increase reliability and result in a greener footprint (due to $\mathrm{CO} 2$ displacement) - especially during unscheduled events like long duration grid outages or loss of connection due to cable failures [14].

However before a GFM WPP can pickup onshore block load for grid restoration, there are different target states and associated demanding transients that it must deal with while maintaining stable voltage and frequency. These include selfstart of WTs and self-sustained houseload operation, followed by magnetization of transformers and charging of offshore cables, ultimately ending with the export link energization as explained in detail in [14]. These stages are of more concern to the WPP operator and stable CIO must be ensured before providing any onshore blackstart service. Thus, hereinafter the energization up to the onshore terminal as shown in Fig. 1. shall be referred to as greenstart to differentiate from the traditionally used 'blackstart' of the main grid.

Recently Scottish Power Renewables in collaboration with Siemens-Gamesa Renewable Energy and Iberdrola Renewables has successfully demonstrated - for the first time - the ability of GFM WTs to operate in island condition supplying local loads while supporting conventional currentcontrol WTs and ultimately provide blackstart service to the grid by energizing the transmission network [15]. That being said, the protection and control systems need to be redesigned and adapted for suitable islanding and blackstart operation to ensure resilient and autonomous startup post blackout [16]. Although controlled voltage ramp-up by the WTs allows for 


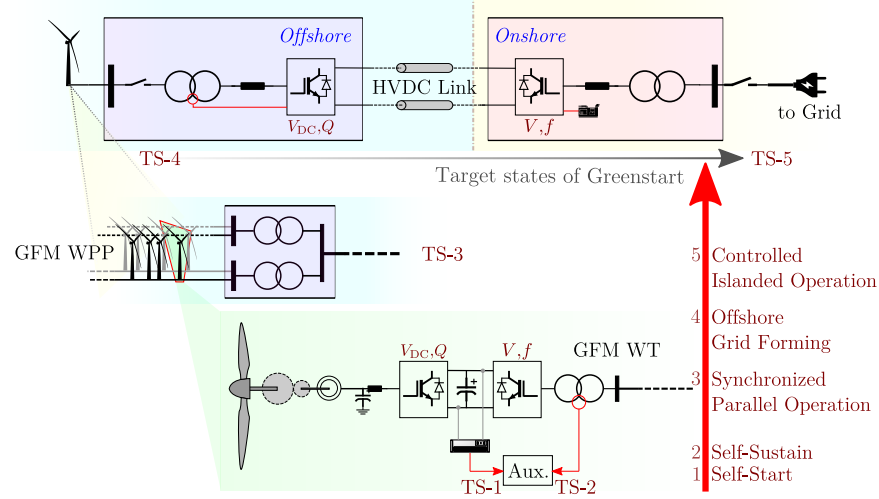

Fig. 1: Target states in the greenstart energization sequence of an HVDC-connected offshore WPP, reproduced from [14].

'soft'-start of the entire offshore network with reduced inrush effects, direct on-line (DOL) or sequential 'hard'-switching increases available fault current to protection schemes and offers enhanced robustness. However, transformers draw significant inrush currents which must be limited to prevent the converter from tripping. This is especially important for the first turbine connection as subsequent energizations are easier because more WTs are up and running [15].

The most straightforward method to limit inrush currents during DOL energization is a controlled reduction in generator voltage as demonstrated in [17]. Alternatively, a preinsertion resistor (PIR) is also commonly used for larger HVDC transformer on the offshore substation and is bypassed after a certain time as shown in recent studies on greenstart of an HVDC-connected offshore WPP [18]. This paper takes inspiration from microgrid converter control that uses a virtual impedance loop to achieve controlled power injection by the distributed generator when it initiates its energy generation [19], [20]. The GFM control of the WT converter can similarly be modified to emulate a virtual resistance $\left(R_{\mathrm{v}}\right)$ at start-up to reduce the terminal voltage during the transformer connection, mimicking the presence of a real PIR and thus limiting inrush effects. However, the DC link of the WT converter interface takes the brunt of the transient and needs to be controlled to maintain converter controllability as described in Sec. III-A

The next section introduces the concept of virtual resistance in converter control followed by a description of the WT model with the control that has been used in this study. After that the transformer energization of a single islanded GFM WT is shown in Sec. IV-A and comparison has been made between DOL, PIR and $R_{\mathrm{v}}$ methods. Then the sensitivity of the inrush transients to the $R_{\mathrm{v}}$ parameters is analysed through PSCAD simulations in Sec. IV-B The impact of $R_{\mathrm{v}}$ on inrush and sympathetic interaction from downstream transformers during string energization has also been studied in Sec. IV-C Finally the feasibility of using $R_{\mathrm{v}}$ for offshore network energization by a GFM WPP is investigated and compared to results for a PIR in Sec. IV-D before the concluding remarks.

\section{VirTual Resistance}

A significant amount of research has been done in tackling the challenges of integrating different technologies of power-electronics, telecommunications, generation and energy storage into microgrids. State-of-art hierarchical multilevel control as proposed in [19], endows flexibility and smartness to microgrid operation by using converters as active thinking and data-processing components with cutting edge functionalities like fault-tolerance, load-sharing, soft-start, island-detection, and smooth mode-switching [20].

The primary level in microgrid hierarchical control is droop based and mainly used to mimic communication-free power sharing in synchronous generators. This also ensures a stable and damped system with energy balance between generation units and energy storage elements [19]. However, resistive line impedance at low voltage applications and inner loop dependent output impedance of converters can negatively affect power sharing. Thus a virtual output impedance loop characterized by Eq. 11, is typically added to emulate synchronous generator's physical inductive behaviour as shown in Fig. 2

$$
v_{\mathrm{o}}=v_{\text {ref }}-i_{\mathrm{o}} Z_{\mathrm{v}} \text { for } Z_{\mathrm{v}} \gg Z_{\mathrm{o}}
$$

Additionally, the virtual impedance loop provides features like reactive-current sharing, harmonic-load compensation and hotswap operation, without any loss in efficiency [19], [20].

The virtual impedance loop can also be used when the distributed generation unit is connected to the microgrid as small differences in voltage phase and/or amplitude can result in overcurrent spikes that can potentially damage the unit [19]. Moreover when the generator begins injecting power, unfavourable transient disturbances can result if the start-up procedure is improper [20].

Large fixed speed wind turbines used external resistors and thyristors to temporarily increase the output impedance of the generator and smoothly reduce inrush transients [19]. Since the virtual impedance can be chosen arbitrarily, it is possible to mimic such a physical soft-starter by setting a high value $R_{\mathrm{i}}$ at the start and gradually reducing it to $\left(R_{\mathrm{f}}=\right) 0$ with a time constant $T$. Such virtual resistance $R_{\mathrm{v}}$ as characterized by Eq. 2 [19], [20], can reduce the transformer energization inrush transient during DOL connection at $t_{\mathrm{o}}$ and by virtue of it being programmed into the GFM converter, help elimiate the need of a physical (fixed value) PIR.

$$
R_{\mathrm{v}}= \begin{cases}0 & \text { if } t<t_{\mathrm{o}} \\ R_{\mathrm{f}}-\left(R_{\mathrm{f}}-R_{\mathrm{i}}\right) e^{-\frac{t-t_{\mathrm{o}}}{T}} & \text { if } t \geq t_{\mathrm{o}}\end{cases}
$$

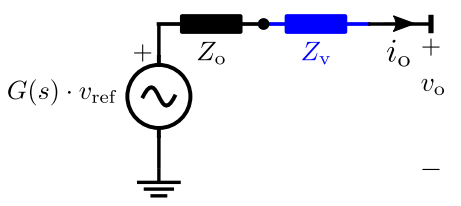

Fig. 2: Thévenin equivalent circuit of converter with virtual impedance loop, reproduced from [19]. This consists of controlled voltage source $G(s) \cdot v_{\text {ref }}$ with $G(s)$ as the closedloop voltage gain transfer function, connected to the grid through the closed loop output impedance $Z_{\mathrm{o}}$ and the virtual impedance $Z_{\mathrm{v}}$. 


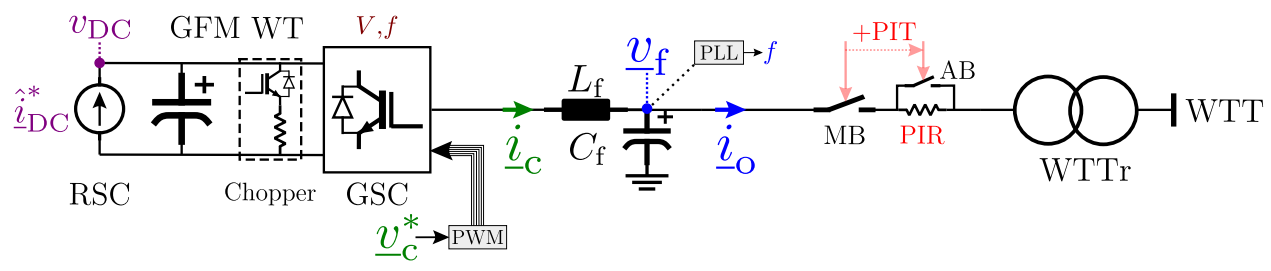

Fig. 3: Model of the islanded open-circuit GFM WT used in this study consisting of: rotor-side-converter (RSC) average model with DC voltage control, DC link chopper, grid-side-converter (GSC) switching model with GFM control, PIR bypassed after PIT using coordinated main and auxiliary breakers (MB, AB), and WT transformer (WTTr) with inrush and saturation.

\section{SyStem MOdEL AND CONTROL}

The schematic of the 8 MW GFM WT under study is shown in Fig. 3. The EMT model developed in PSCAD consists of average model of rotor-side-converter (RSC) and detailed switching model of the grid-side-converter (GSC) with a chopper at the DC link. The GSC terminal is connected to the WT transformer (WTTr) through the main breaker (MB) for DOL energization. A PIR is also present for inrush limitation that is bypassed by closing the auxiliary breaker (AB) after a certain PIT. There is no load connected at the WT terminal (WTT) i.e. the GFM WT operates in islanded mode open circuit as this study focuses on the transients during no-load transformer energization. The WTTr is modelled with inrush and saturation characteristics as shown in Fig. 12 in Appendix A. The values of all the circuit parameters are based on [8], [18] and tabulated in Table [ in the Appendix A

\section{A. Control and tuning}

The GSC is a 2-level voltage-sourced-converter (VSC) switching at $2 \mathrm{kHz}$ with GFM strategy based on improved direct power control [21] as shown in Fig. 4a and similar to the implementation in [8]. The controller is tuned according to criteria as described in detail in [8] equivalent to a slow current controller with natural frequency of $4 \mathrm{~Hz}$ and damping ratio of

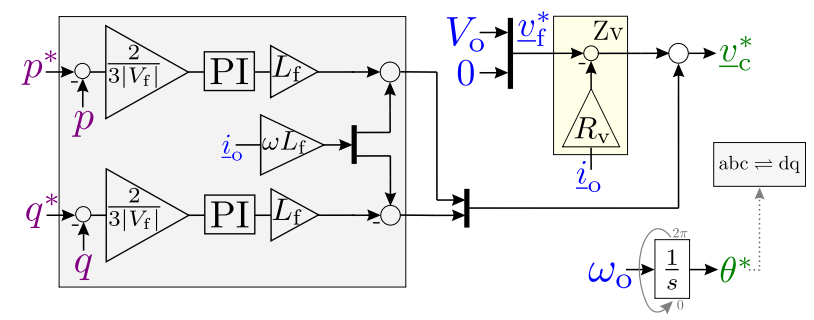

(a) Direct Power GFM control for GSC based on [8]. It consists of real and reactive power controllers for load sharing. The voltage reference is given in place of the grid voltage magnitude [21] and a virtual phase angle is used in place of PLL-generated voltage phase angle [22]. Virtual impedance (Zv) loop has been added for the study based on Eq. 1

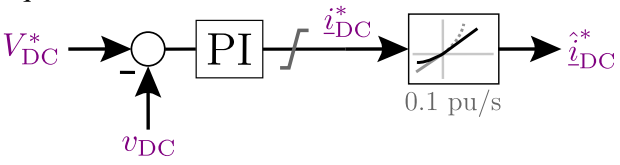

(b) DC link voltage controller that generates reference for RSC current source.

Fig. 4: Control for GSC and RSC of GFM WT.
0.74. The virtual impedance loop has been added in the GFM control structure based on Eq. 1 for studying the energization transient using virtual resistance as shown in Fig. $4 \mathrm{a}$

Since the GSC controls the terminal AC voltage of the WT, the RSC is tasked with controlling the WT DC link voltage. This is modelled with a current source that receives its reference from a DC link voltage controller as shown in Fig. 4b The control loop is tuned for $3 \mathrm{~Hz}$ bandwidth [9] and $55^{\circ}$ phase margin for stable operation. Although optimal control to maximize generator output power ramp rate has been proposed in [9], a conservative limit of $0.1 \mathrm{pu} / \mathrm{s}$ has been used here as a faster ramp-up can lead to vibrations in the shaft and excite tower/blade oscillatory modes. The tuned values of the control loops are tabulated in Table $\Pi$ in the Appendix.

\section{B. Assumptions}

Contrary to grid-following WT, GSC cannot control the DC link in GFM mode by ensuring power balance as the power flow is now set by the AC load and not the turbine controller, which conventionally extracts maximum power from the wind. However, now it has to regulate the speed - especially avoid over-speeding during low AC load and high winds. Thus, RSC is required to maintain constant DC link voltage by ensuring that the generator output power tracks the load power demand. This can be achieved by optimally controlling the generator speed based on the WT power-speed characteristics [9]. Such a DC link control has much lower bandwidth (maximum $5 \mathrm{~Hz}$ [9]) than the inner speed and torque/current control loops, and has been shown to perform well with changing wind speeds and for seamless transition between weak and strong grid connection modes. Thus, it has been adapted with some simplification as shown in Fig. $4 \mathrm{~b}$, since the mechanical dynamics are not in the scope of this study.

Moreover, the redesign of the turbine speed controller as discussed in [11], allows for stable operation over an extended operational range (including strongly de-rated and negative power values), cope with sudden changes in generator torque during large load steps, and prevent transient over-speeding of the WT during load disconnections in weak grid conditions.

\section{Simulation Study and Results}

Most modern WTs rely on an internal backup UPS for auxiliary power during idling operation to supply house-load [14]. Moreover, energy storage required to handle the transient load variations has been shown to be acceptable with the potential to be reduced further using advanced technologies in future 
[9]. Additionally, pre-charging control has been proposed in [10] which allows charge retention on the DC bus following a blackout, thus enabling terminal voltage build-up for network energization and load pick-up, if wind energy is available. A synchrophasor-enabled algorithm is also proposed in [10] for autonomous synchronization and 'hot-swap' operation without resetting of controller dynamic states or requirement of energy storage.

With the above in consideration, the energization sequence for this study consists of the following three steps:

1) Pre-charging - a controlled DC link voltage is necessary for GSC to operate. This is regulated by the RSC as described in Section III-A using the control loop shown in Fig. $4 \mathrm{~b}$ The power ramp-rate limit is disabled during this stage as it is assumed that proper control such as that presented in [10] allows charge retention on DC bus after a blackout, or the backup UPS is sufficient to sustain the auxiliary requirements of the WT for a critical period of time. In the worst case, the DC voltage would be built up by first spinning the generator up to a minimum voltage and then deblocking the RSC (operating the switches for rectification with backup power from UPS) to charge the DC link capacitor, similar to the AC side pre-charging of the offshore MMC as shown in [18] (Stage 2). This is however out of the scope of this study.

2) Grid forming - once the DC link voltage is controlled, the GSC can be deblocked in GFM mode to control the $\mathrm{AC}$ voltage and frequency at the filter output.

3) Connection - after the DC bus initialization by RSC and terminal AC voltage and frequency control by GSC, the WTTr is connected by closing MB. The inrush transients during energization of the WTTr are the main focus of this study.

\section{A. Proof-of-concept}

In this section the inrush transient and its associated DC link dynamics along with the impact on AC terminal voltage are compared for three energization methods namely, direct online (DOL), using PIR that is bypassed by closing $A B$ after PIT delay, and implementing a virtual resistance $\left(R_{\mathrm{v}}\right)$ in the GFM control of GSC. For DOL and $R_{\mathrm{v}}$ methods AB is closed together with MB to bypass the PIR from start. Figure 5 shows a comparison of the results for DOL, PIR and $R_{\mathrm{v}}$ methods when WTTr is connected at $2 \mathrm{~s}$.

The DOL energization is shown here as the base case scenario. The values for the PIR case and $R_{\mathrm{v}}$ methods has been chosen only as an example to demonstrate that the virtual resistance emulation can indeed reduce inrush transient and its associated dynamics. PIR/PIT and $R_{\mathrm{v}}$ parameter values are chosen to result in about similar inrush current peak (about $0.5 \mathrm{pu})$ as shown in Fig. 5b Thus, PIR and $R_{\mathrm{i}}$ of $0.05 \Omega$ $(0.8 \mathrm{pu})$ are selected with $T$ set to $0.04 \mathrm{~s}$, so that the time when $R_{\mathrm{v}}$ settles to zero $(=5 T)$ is equal to the PIR bypass time (=PIT) of $0.2 \mathrm{~s}$.

From Fig. $5 \mathrm{a}$ it can be seen that $R_{\mathrm{v}}$ reduces the transient real and reactive power peaks to $0.11 \mathrm{MW}$ and $0.93 \mathrm{MVar}$ compared to $0.42 \mathrm{MW}$ (2.87 MVar) and 0.37 MW (0.7 MVar)
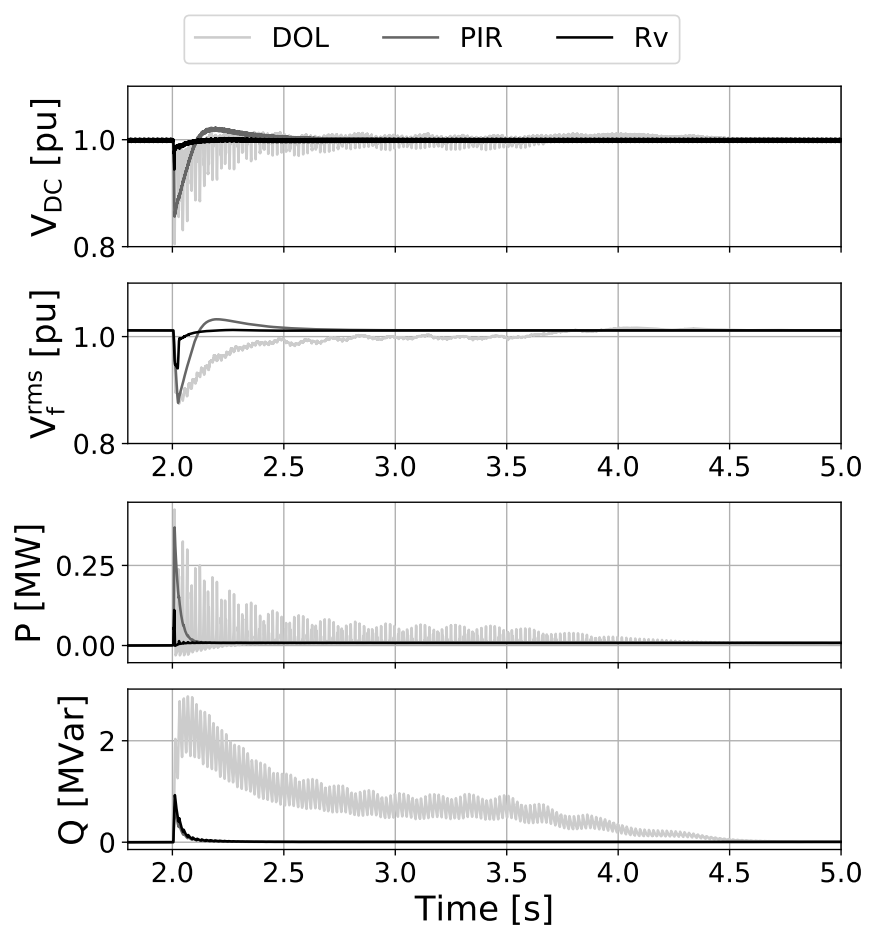

(a) DC link dynamics and impact on the output RMS AC voltage of GSC along with real and reactive power outputs of WT.
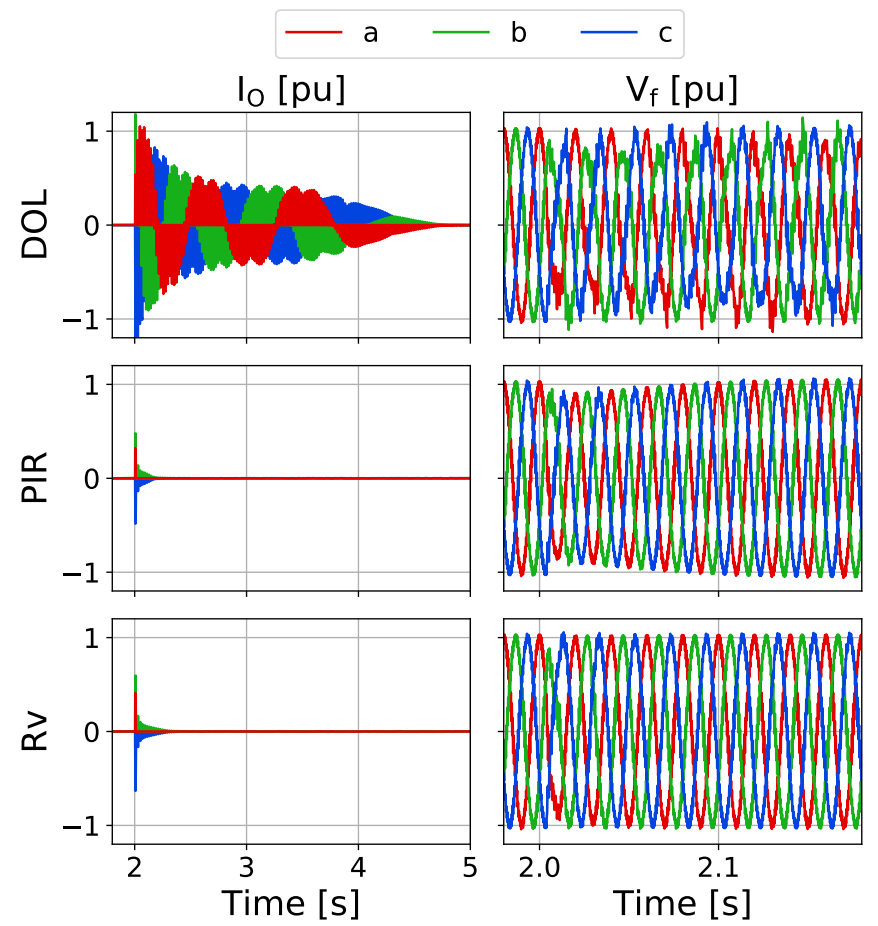

(b) 3-phase instantaneous currents and voltage output of GSC.

Fig. 5: Comparison of waveforms during inrush transient for DOL, PIR and $R_{\mathrm{v}}$ energization of WTTr. 
for DOL and PIR cases, respectively. This is corroborated by Fig. 5b, clearly demonstrating that $R_{\mathrm{v}}$ reduces the inrush current/power transient significantly — both in peak amplitude and settling time - similar to the PIR case. Additionally, the associated DC link voltage drop is lesser for $R_{\mathrm{v}}$ as it dips to $0.81 \mathrm{pu}, 0.86 \mathrm{pu}$ and $0.95 \mathrm{pu}$ for DOL, PIR and $R_{\mathrm{v}}$ methods, respectively as shown in Fig. 5a. Moreover while the RMS AC voltage dip is almost identical for DOL and PIR cases $(0.87 \mathrm{pu})$, it is smaller for $R_{\mathrm{v}}(0.94 \mathrm{pu})$. Lastly it can also be observed from Fig. $5 \mathrm{~b}$ that while both PIR and $R_{\mathrm{v}}$ reduce distortion in the 3-phase instantaneous $\mathrm{AC}$ voltages, the transient dip is recovers faster for $R_{\mathrm{v}}$.

\section{B. Sensitivity analysis}

Since the parameters in Sec. IV-A were chosen to only demonstrate the concept, a sensitivity analysis has been done to study how the inrush transients vary with different values of the $R_{\mathrm{v}}$ parameters. This can give some insight into how to choose the virtual resistance parameters. For this study the values of $R_{\mathrm{i}}$ used are $0.05 \mathrm{pu}, 0.5 \mathrm{pu}$ and $1.5 \mathrm{pu}$, and for $T$ are $0.05 \mathrm{~s}, 0.2 \mathrm{~s}$ and $1.0 \mathrm{~s}$. The results of the effect of varying $R_{\mathrm{i}}$ and $T$ on the real and reactive power outputs of the WT, DC link voltage and the 3-phase RMS AC voltage output of WT are presented in Fig. 6a The effect on the inrush currents is shown in Fig. $6 \mathrm{~b}$.

As expected, Figs. 6a and 6b show that the transient power and current peaks decrease as $R_{\mathrm{i}}$ is increased. Moreoever, Fig. 6a also shows that larger values of $R_{\mathrm{i}}$ help reduce the $\mathrm{AC}$ and DC voltage dips. Additionally, it can be inferred from Fig. 6a that the transient settling time and voltage dip recovery time increases for smaller values of $T$, especially at lower $R_{\mathrm{i}}$. This is also corroborated by Fig. 6b which shows that the inrush current transient damps faster for larger values of $T-$ the effect being more visible for smaller $R_{\mathrm{i}}$.

\section{String energization}

Once the WTTr of the GFM WT has been energized, the next step is to energize the WTTrs of the other WTs connected in the string, as shown in Fig. 7 In this section the effect of using virtual resistance to reduce inrush transients of downstream WTTrs in the string has been studied. In the previous energization studies we assume complete WTTr deenergization for best case residual flux $\left(\psi_{\text {res }}=0\right)$. However, in this section sympathetic interaction between series and parallel connected WTTrs [23] has also been studied by simulating for worst case residual flux $(70 \%)-$ setting $\psi_{\text {res }, \mathrm{a}}=0.7 \mathrm{pu}$, $\psi_{\text {res }, \mathrm{b}}=0 \mathrm{pu}$ and $\psi_{\text {res }, \mathrm{c}}=-0.7 \mathrm{pu}$ in the WTTr PSCAD model [17]. BRK1 is closed at $2 \mathrm{~s}$ to energize Tr-1, BRK2 at $6 \mathrm{~s}$ to energize $\operatorname{Tr}-2$ and finally BRK3 at $10 \mathrm{~s}$ to energize Tr- 3 . The value of $R_{\mathrm{i}}=1 \mathrm{pu}$ and $T=0.06 \mathrm{~s}$ has been used for each energization event but may be different for the subsequent WTTr connections.

It is clear from Fig. 9a that virtual resistance helps reduce the transient power peaks and voltage dips for both best and worst case residual flux. Moreover, from the instantaneous current waveforms in Fig. $9 \mathrm{~b}$ it can be inferred that although $R_{\mathrm{v}}$ helps reduce the Tr-1 inrush significantly both in peak

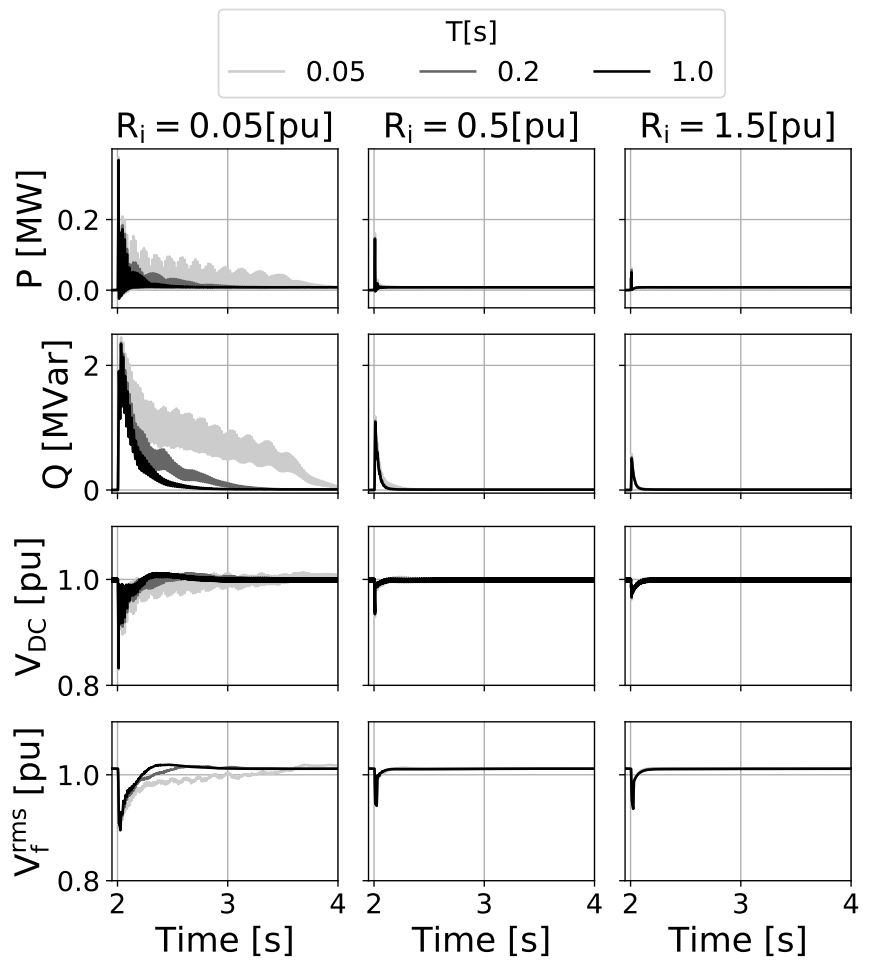

(a) Real and reactive power output of WT with associated DC link dynamics and impact on the output RMS AC voltage of GSC.

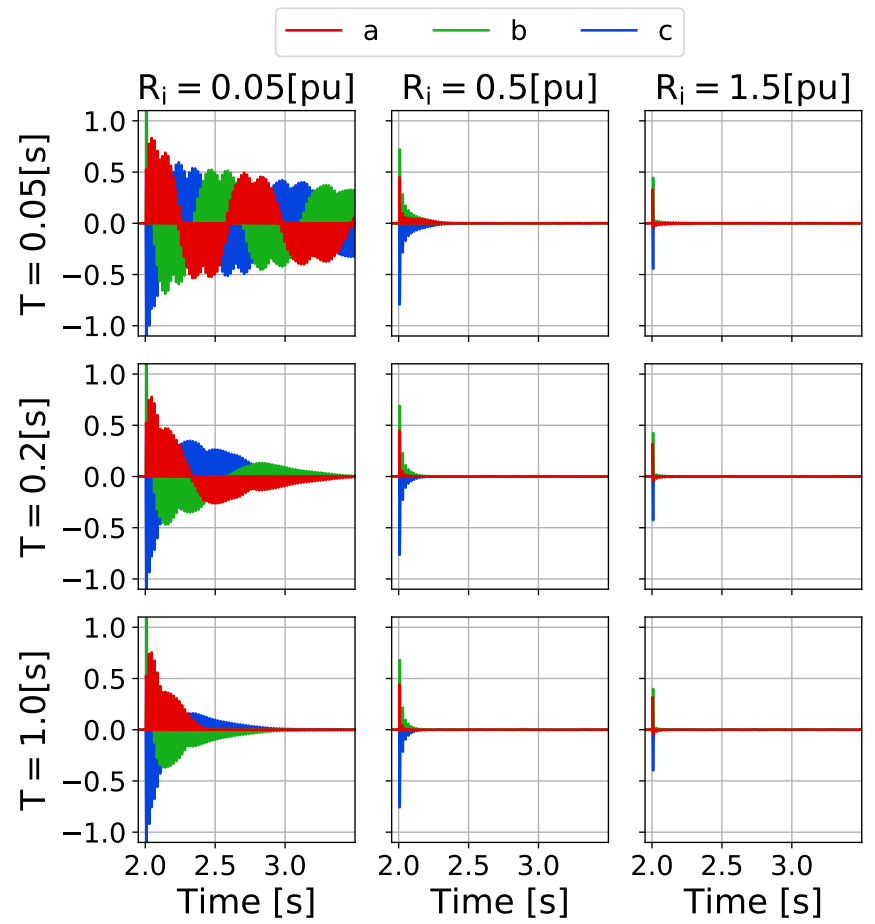

(b) 3-phase instantaneous AC current output of GSC: effect of increasing $R_{\mathrm{i}}$ is shown from left to right (horizontal) and that of increasing $T$ is shown from top to bottom (vertical).

Fig. 6: Waveforms during $R_{\mathrm{v}}$ energization of WTTr: sensitivity to $R_{\mathrm{i}}=0.05 \mathrm{pu}, 0.5 \mathrm{pu}$ and $1.5 \mathrm{pu}$, and $T=0.05 \mathrm{~s}, 0.2 \mathrm{~s}$ and $1.0 \mathrm{~s}$. 


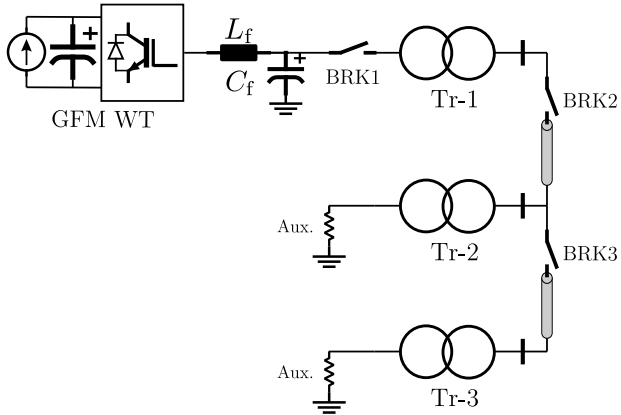

Fig. 7: Model of the string to study energization of downstream WTTrs by GFM WT.

amplitude and duration, only a reduction in peak is seen for the subsequent WTTrs (Tr-2 and Tr-3) with no impact on the duration of decay of the sympathetic inrush, especially for worst case residual flux.

\section{WPP level energization}

In this section the virtual resistance has been implemented in the GFM WTs of a $400 \mathrm{MW}$ HVDC-connected WPP for the energization of its 400 MVA offshore HVDC transformer (HVDC-Tr), based on that in [18] and with aggregation as shown in Fig. 8 . Figure 10 depicts the energization of the offshore HVDC-Tr at $1.3 \mathrm{~s}$ by the GFM WPP using a PIR versus $R_{\mathrm{v}}$. The $120 \Omega$ PIR, bypassed after $0.3 \mathrm{~s}$ PIT [18], is used as base case. $R_{\mathrm{i}}=4 \mathrm{pu}$ and $T=0.06 \mathrm{~s}$ have been used in the $R_{\mathrm{v}}$ case to achieve a similar transient current peak $(0.5 \mathrm{pu})$ and settling time $(5 T=$ PIT) as in the PIR case as shown in Fig. $10 \mathrm{a}$.

Transients in WPP active and reactive power output, and in RMS AC voltage at the WPP and PCC-2 terminals are shown in Fig. $10 \mathrm{~b}$ for both cases. It is clear that $R_{\mathrm{v}}$ effectively limits the inrush transient peak by decreasing the voltage in a smooth manner like in the case of a physical soft-starter. Moreover, there are no second transient power peaks in the $R_{\mathrm{v}}$ case as opposed to when the PIR is bypassed at $1.6 \mathrm{~s}$.

In the PIR case the main voltage drop occurs between the WPP and PCC-2 terminals (i.e. across the PIR), which is reflected by the relatively small variation in the WPP terminal voltage in Fig. 10b. In the $R_{\mathrm{v}}$ case in contrast, the main voltage drop can be said to occur (virtually) through $R_{\mathrm{v}}$ i.e. inside the GFM WPP acting as a voltage sources (as shown in Fig. 2), which is reflected by the significant drop in the the WPP terminal voltage in Figs. 10b (RMS) and 10c (3phase instantaneous). This can be corroborated by calculating

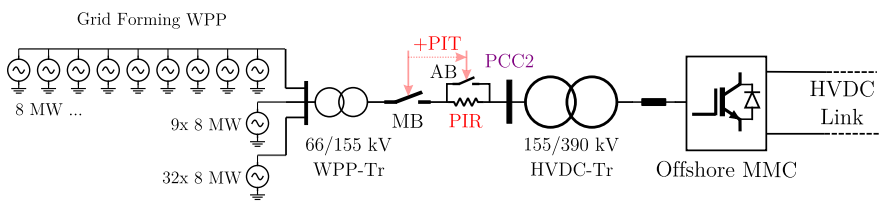

Fig. 8: Model of the GFM WPP with average model WTs used to study the offshore HVDC transformer (HVDC-Tr) inrush transients using PIR and $R_{\mathrm{v}}$.

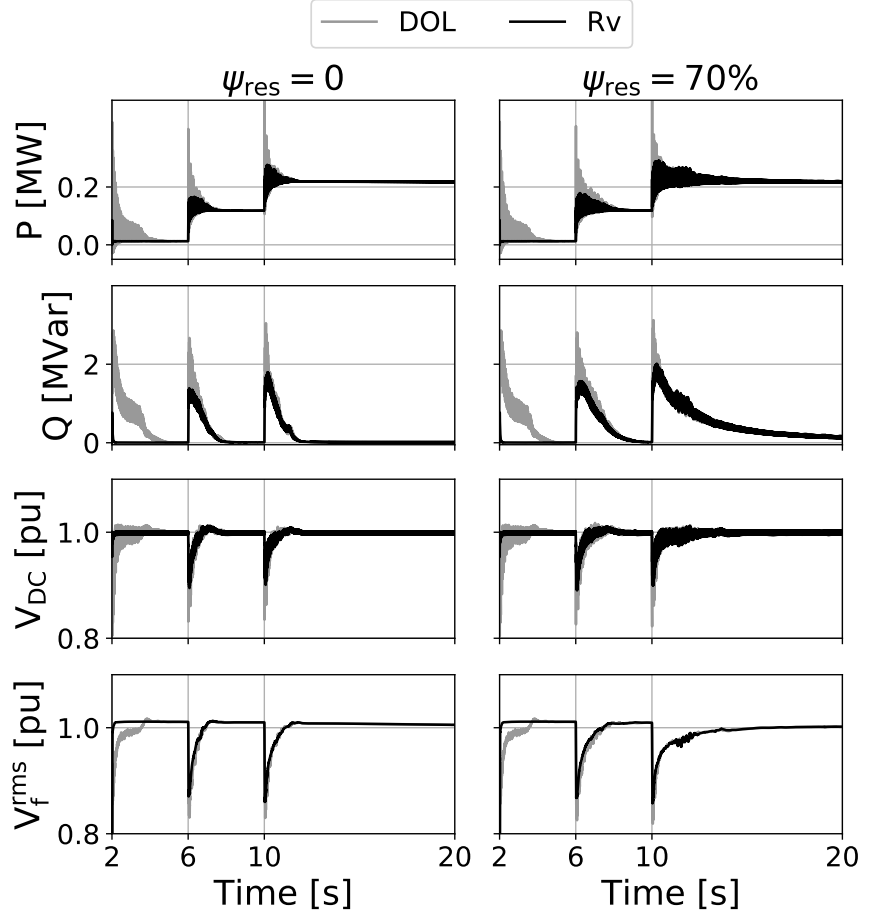

(a) Real and reactive power output of GFM WT and associated DC link voltage and output RMS AC voltage dynamics.
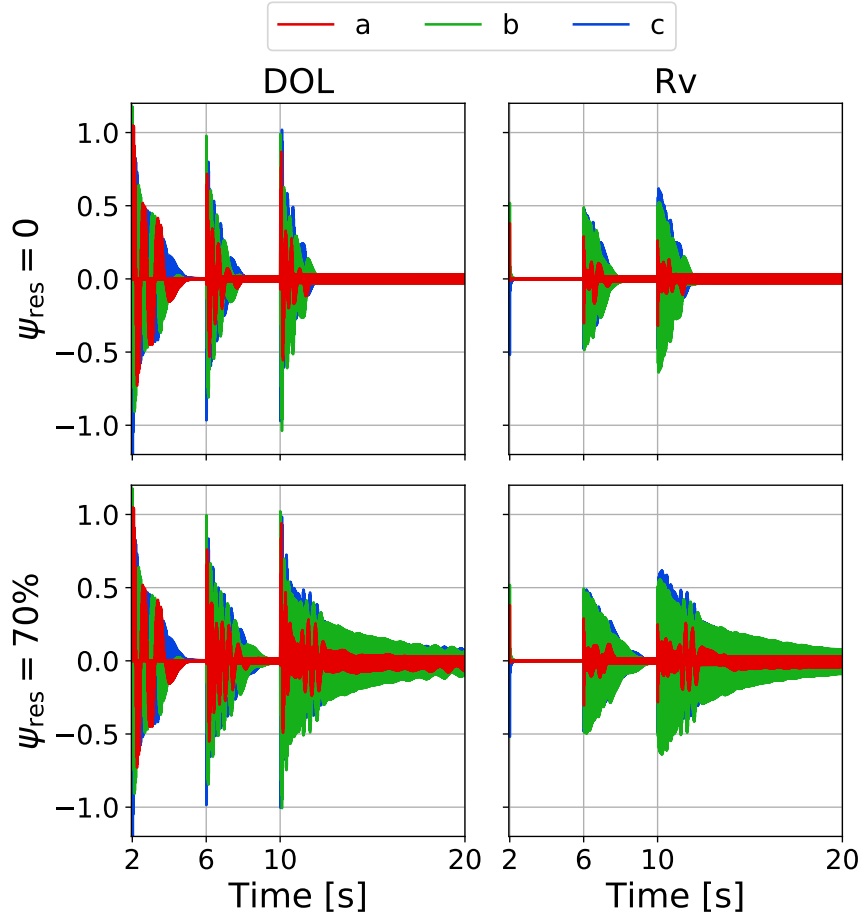

(b) 3-phase instantaneous WT output currents.

Fig. 9: Waveforms during DOL and $R_{\mathrm{v}}$ energization of downstream WTTrs in string, for best $\left(\psi_{\text {res }}=0\right)$ and worst $\left(\psi_{\text {res }}=70 \%\right)$ case residual flux. 


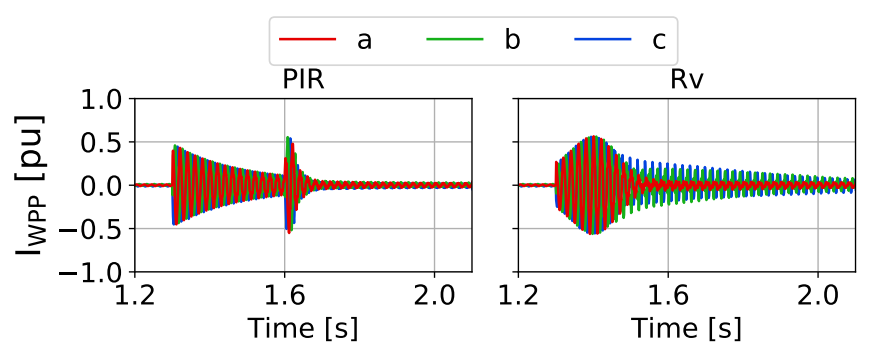

(a) 3-phase instantaneous output current of WPP.
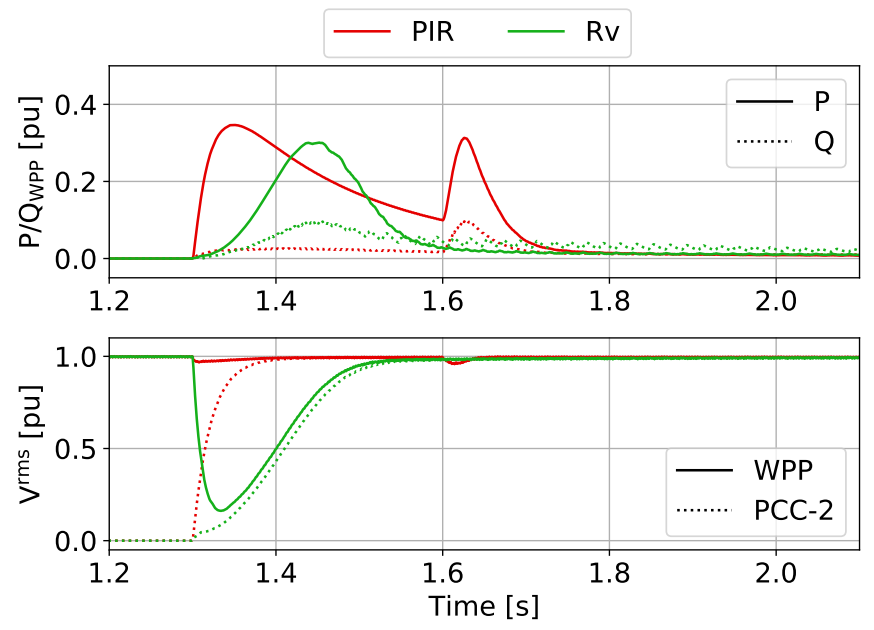

(b) Active and reactive power output of WPP and 3-phase RMS AC voltage at WPP and offshore PCC-2 terminal.
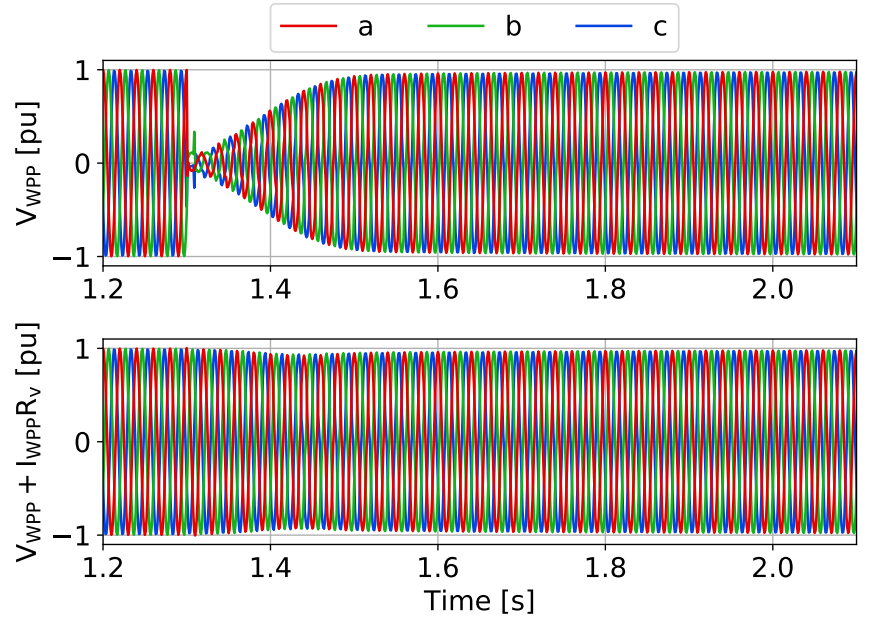

(c) 3-phase instantaneous $\mathrm{AC}$ voltage for $R_{\mathrm{v}}$ case: the top signal is measured at WPP terminal while the bottom signal is the calculated (virtual) GFM voltage at WPP terminal without $R_{\mathrm{v}}$ drop.

Fig. 10: Offshore HVDC-Tr energization at $1.3 \mathrm{~s}$ by GFM WPP using a $120 \Omega$ PIR with $0.3 \mathrm{~s}$ PIT vs using $R_{\mathrm{v}}$ with $R_{\mathrm{i}}=4 \mathrm{pu}, T=0.06 \mathrm{~s}$.

the (virtual) voltage drop across $R_{\mathrm{v}}$ and removing it from the WPP terminal voltage response $\left(V_{\mathrm{WPP}}+I_{\mathrm{WPP}} R_{\mathrm{v}}\right)$ as shown in Fig. 10c.

It can be inferred from Fig. 10c that although $R_{\mathrm{v}}$ is able to limit the inrush current transient to a peak similar to that of the PIR case (Fig. 10a), it does so at the expense of the

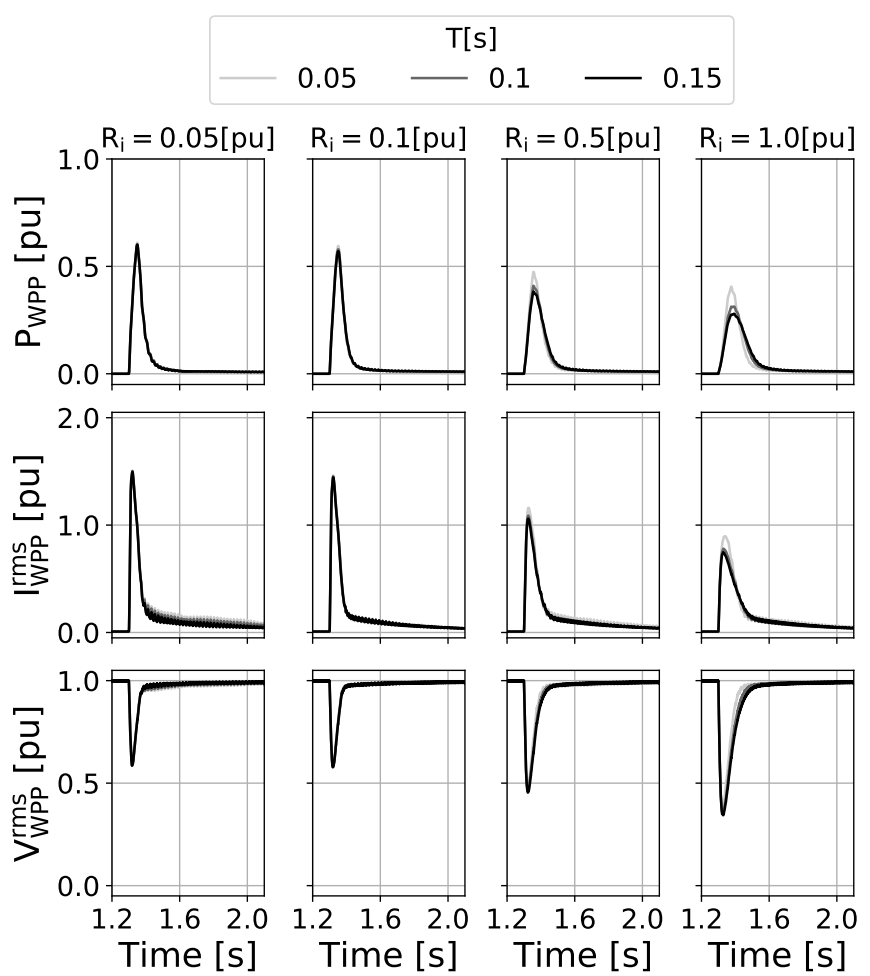

Fig. 11: Sensitivity analysis of real power and 3-phase RMS AC current and voltage output of WPP during offshore HVDCTr energization at $1.3 \mathrm{~s}$ for different values of $R_{\mathrm{v}}$.

WPP terminal voltage, which can drop significantly - to levels that can be as low as those characteristic of faults. Fault clearing during energization is dependent on undervoltage trip relays due to the low short circuit levels and thus, the application of the $R_{\mathrm{v}}$ method to the energization of $\mathrm{HV}$ transformers may require different protection settings. As the bottom plot in Fig. 10c moreover suggests, some of the protection changes could rely instead on a virtual voltage calculated using the given $R_{\mathrm{v}}$ and local voltage and current measurements. Additionally, different $R_{\mathrm{v}}$ initial values and time constants can be used for different resulting voltage dips and transient current/power peaks as shown in Fig. 11 Likewise, other time characteristics/functions (i.e. different from that of Eq. 2) may also be used for $R_{\mathrm{v}}$.

\section{CONClusion}

In this paper virtual resistance has been implemented in the wind turbine converter control during energization of its transformer. This helps avoid using a pre-insertion resistor during sequential hard-switching and without any loss of fault selectivity as in the soft-start case. However, the brunt of the transient is borne by the wind turbine DC link and thus, rotor-side control is essential for governing the dynamics. Simulation results show that the inrush transients during open circuit energization of transformer can be reduced similar to using a pre-insertion resistor. Moreover, the sensitivity analysis gives insight into how the virtual resistance value impacts the transients in current/power output and DC/AC voltage of 
the wind turbine. Additionally, the study on the energization of downstream transformers in a string by a grid forming wind turbine demonstrates that virtual resistance can reduce transient current peak for both best and worst case residual flux. However, it is important to note that worst case residual flux leads to significant sympathetic interaction lasting for a sustained period of time, with virtual resistance effective only in reducing the peak amplitude. Finally at the wind power plant level, the virtual resistance method can also be used to minimize the inrush transient during the large offshore transformer energization. However, protection settings need to be changed to avoid the voltage dip triggering under-voltage trip relays.

\section{APPENDIX A}

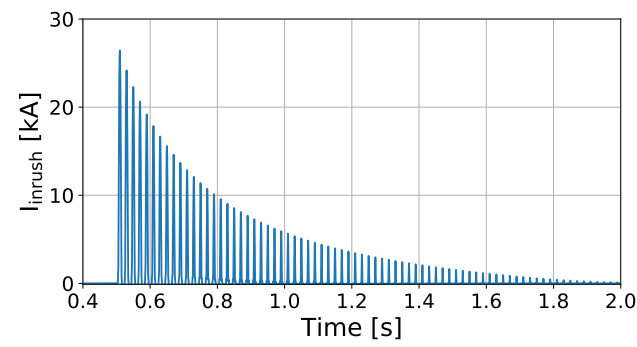

Fig. 12: Inrush current characteristic of $8 \mathrm{MVA}$ WTTr during open circuit energization by an ideal $0.69 \mathrm{kV}$ voltage source.

TABLE I: Main circuit parameters of the model.

\begin{tabular}{ll}
\hline Parameter & Value \\
\hline WT Rating & $8 \mathrm{MW}$ \\
WTTr & $0.69 / 66 \mathrm{kV}, X=0.1 \mathrm{pu}$ \\
WT GSC Filter & $L_{\mathrm{f}}=10 \%, C_{\mathrm{f}}=5 \%$ \\
WT GSC Switching frequency & $2 \mathrm{kHz}$ \\
WT DC link & $1.45 \mathrm{kV}, C_{\mathrm{DC}}=30000 \mu \mathrm{F}$ \\
\hline
\end{tabular}

TABLE II: Tuned controller values in pu.

\begin{tabular}{llll}
\hline Scope & Control & Parameter & Value \\
\hline GSC & Current control & P & 0.4 \\
& Voltage control & PI & $0.02,2.55$ \\
& PLL & PI & 10,25 \\
& Frequency control & $k_{\mathrm{f}}$ & 50 \\
RSC & DC voltage control & PI & $0.09,0.92$ \\
& Absolute limit & Min., Max. & \pm 1.1 \\
& Power ramp-rate limit & Up, Down & $\pm 0.1 \mathrm{~s}^{-1}$ \\
\hline
\end{tabular}

\section{ACKNOWLEDGMENT}

The authors gratefully acknowledge Asger B. Abrahmsen for discussions leading up to this work.

\section{REFERENCES}

[1] International Energy Agency (IEA), "Renewables 2020: Analysis and forecast to 2025," 2020 .

[2] S. De Boeck, D. Van Hertem, K. Das, P. E. Sørensen, V. Trovato, J. Turunen, and M. Halat, "Review of Defence Plans in Europe: Current Status, Strengths and Opportunities," CIGRE Transactions on Science \& Engineering, vol. 5, pp. 6-16, 2016.
[3] M. N. I. Sarkar, L. G. Meegahapola, and M. Datta, "Reactive Power Management in Renewable Rich Power Grids: A Review of GridCodes, Renewable Generators, Support Devices, Control Strategies and Optimization Algorithms," IEEE Access, vol. 6, pp. 41458-41489, 2018.

[4] Australian Energy Market Operator (AEMO), "Black system South Australia 28 September 2016 - Final Report," 2017.

[5] National Grid ESO, "Technical Report on the events of 9 August 2019," Sep 2019.

[6] Elia - National Control Center \& Market Development, "Study on the Review of the Black Start Ancillary Services," 2018.

[7] National Grid ESO, "Black Start from Non - Traditional Generation Technologies," 2019.

[8] A. Jain, J. N. Sakamuri, and N. A. Cutululis, "Grid-forming control strategies for black start by offshore wind power plants," Wind Energy Science, vol. 5, no. 4, pp. 1297-1313, 2020.

[9] X. Yuan, F. Wang, D. Boroyevich, Y. Li, and R. Burgos, "DC-link Voltage Control of Full Power Converter for Wind Generator operating in Weak Grid Systems," IEEE Transactions on Power Electronics, vol. 24, no. 9, pp. 2178-2192, 2009.

[10] P. M. Farsani, S. G. Vennelaganti, and N. R. Chaudhuri, "Synchrophasorenabled power grid restoration with DFIG-based wind farms and VSCHVDC transmission system," IET Generation, Transmission \& Distribution, vol. 12, no. 6, pp. 1339-1345, 2017.

[11] M. Shan, W. Shan, F. Welck, and D. Duckwitz, "Design and laboratory test of black-start control mode for wind turbines," Wind Energy, vol. 23, no. 3, pp. 763-778, 2020.

[12] J. N. Sakamuri, Ö. Göksu, A. Bidadfar, O. Saborío-Romano, A. Jain, and N. A. Cutululis, "Black Start by HVdc-connected Offshore Wind Power Plants," in IECON 2019 - 45th Annual Conference of the IEEE Industrial Electronics Society, Lisbon, 2019.

[13] A. Jain, J. N. Sakamuri, K. Das, Ö. Göksu, and N. A. Cutululis, "Functional Requirements for Blackstart and Power System Restoration from Wind Power Plants," in 2nd International Conference on LargeScale Grid Integration of Renewable Energy in India. New Delhi: Energynautics GmbH, 2019.

[14] A. Jain, K. Das, Ö. Göksu, and N. A. Cutululis, "Control Solutions for Blackstart Capability and Islanding Operation of Offshore Wind Power Plants," in 17th International Wind Integration workshop. Stockholm: Energynautics GmbH, 2018.

[15] A. Roscoe, P. Brogan, D. Elliott, T. Knueppel, I. Gutierrez, P. Crolla, R. D. Silva, and Juan-Carlos Perez Campion, "Practical Experience of Providing Enhanced Grid Forming Services from an Onshore Wind Park," in 18th International Wind Integration Workshop. Energynautics $\mathrm{GmbH}, 2019$.

[16] I. Gutierrez, P. Crolla, A. Roscoe, P. Brogan, D. Elliott, T. Knueppel, Juan-Carlos Perez Campion, and R. D. Silva, "Operator Considerations for the Implementation of Testing Enhanced Grid Forming Services on an Onshore Wind Park," in 19th International Wind Integration Workshop. Energynautics GmbH, 2020.

[17] I. Arana, A. Hernandez, G. Thumm, and J. Holboell, "Energization of wind turbine transformers with an auxiliary generator in a large offshore wind farm during islanded operation," IEEE Transactions on Power Delivery, vol. 26, no. 4, pp. 2792-2800, 2011.

[18] A. Jain, O. Saborío-romano, J. N. Sakamuri, and N. A. Cutululis, "Blackstart from HVDC-connected Offshore Wind : Hard vs Soft Energization," IET Renewable Power Generation, vol. 15, no. 1, pp. 127-138, 2021.

[19] J. M. Guerrero, J. C. Vasquez, J. Matas, L. G. De Vicuña, and M. Castilla, "Hierarchical control of droop-controlled AC and DC microgrids - A general approach toward standardization," IEEE Transactions on Industrial Electronics, vol. 58, no. 1, pp. 158-172, 2011.

[20] B. Arbab-Zavar, E. J. Palacios-Garcia, J. C. Vasquez, and J. M. Guerrero, "Smart Inverters for Microgrid Applications: A Review," Energies, vol. 12, no. 5, p. 840, 2019.

[21] Y. Gui, X. Wang, and F. Blaabjerg, "Vector Current Control Derived from Direct Power Control for Grid-Connected Inverters," IEEE Transactions on Power Electronics, vol. 34, no. 9, pp. 9224-9235, 2019.

[22] P. Cheng and H. Nian, "Direct power control of voltage source inverter in a virtual synchronous reference frame during frequency variation and network unbalance," IET Power Electronics, vol. 9, no. 3, pp. 502-511, 2016.

[23] H. Bronzeado and R. Yacamini, "Phenomenon of sympathetic interaction between transformers caused by inrush transients," IEE Proceedings: Science, Measurement and Technology, vol. 142, no. 4, pp. 323-329, 1995. 